\title{
Is the Equality between the Inertial and the Gravitational Mass Empirically Falsifiable?*
}

¿Es falsable la igualdad entre la masa inercial y la gravitacional?

Josu Zabaleta Imaz ${ }^{\dagger}$

\begin{abstract}
Following a suggestion made by Einstein, a non-empirical or structural interpretation of the equality between the inertial and the gravitational mass is proposed. To this aim, I will relate my line of argument regarding the foundations of the General Relativity to an analogous passage on the history of science in Newton's preparatory works for the Principia. In both cases, two concepts of mass are mere expressions of two different frames of reference (inertial for Newton, non-inertial for Einstein). As a result, the ongoing experiments, whose aim is to prove empirically the equality between the inertial and gravitational mass, should not be accepted.
\end{abstract}

Keywords: inertial mass - gravitational mass - equality - non-empirical or structural interpretation - Einstein - Newton

\section{Resumen}

Siguiendo una sugerencia de Einstein, se propone una interpretación no-empírica o estructural de la igualdad entre la masa inercial y la gravitacional. Para ello, relacionaré mi línea argumental sobre los fundamentos de la Relatividad General con un pasaje análogo de la historia de la ciencia en los trabajos preparatorios de Newton para los Principia. En ambos casos, los dos conceptos de masa son meras expresiones de dos marcos de referencia diferentes (inercial para Newton, no inercial para Einstein). En consecuencia, no deben aceptarse los experimentos en curso, cuyo objetivo es demostrar empíricamente la igualdad entre la masa inercial y la gravitatoria.

Palabras clave: masa inercial - masa gravitacional - igualdad - interpretación no-empírica o estructural - Einstein Newton

\footnotetext{
* Received: 21 November 2017. Accepted with revisions: 17 February 2018.

${ }^{+}$University of the Basque Country, Spain. To contact the author, please write to: josu.zabaleta.imaz@gmail.com. Metatheoria 9(1)(2018): 51-61. ISSN 1853-2322. eISSN 1853-2330.

(C) Editorial de la Universidad Nacional de Tres de Febrero. Publicado en la República Argentina.
} 


\section{Introductory remarks ${ }^{1}$}

The equality between the inertial and the gravitational mass (EIGM) is widely considered by scholars to be an "empirical fact" or an empirically falsifiable proposition. According to this view, not only the equality itself, but also the Physical Theory based ${ }^{3}$ on this equality, namely the General Relativity (GR), could be refutable by an experiment. The many experiments which have been undertaken in the last decades in order to refute the EIGM have not succeeded ${ }^{4}$ and, therefore, the validity of the equality remains unchanged.

Nevertheless, the position of the founder of the GR on this issue is diametrically opposed to the empirical interpretation of the EIGM. In his Grundzüge der Relativitätstheorie (1969), Einstein defines the EIGM as an equality whose explanation (Erklärung) is provided by the GR. In this way, says Einstein, the GR does justice to the EIGM by reducing (reduzieren) the numerical equality to an "equality of the Essence" (Gleichheit des Wesens) (Einstein 1969). In other words, according to Einstein, due to the contribution made by the GR, the equality ceases to be a mere empirical fact (Erfahrungstatsache).

In this paper, I will argue in defense of the aforementioned non-empirical interpretation of the EIGM. For this purpose, in the first part of the paper, I will relate the EIGM to a similar episode that occurred in the History of Physics during the $17^{\text {th }}$ century. In particular, I will demonstrate that the relationship between the equality between Newton's two concepts of mass and the inertial frame system is analogous to the relationship between EIGM and the non-inertial frame system in the GR.

If my interpretation of the EIGM comes out to be sustainable, it would not only be inadmissible to speak about a falsifiability or empirical character of the EIGM, but also, the equality itself would be refuted. We would no longer have an equality between two masses, but rather the same mass manifesting itself in two different ways, i.e. in two different frames of reference. ${ }^{5}$ Therefore, the experiments to prove the falsifiability of the EIGM which were designed after $^{6}$ the emergence of the GR, and some of which are ongoing, should be considered as late remainders or examples of the classical or Newtonian view of Physics.

\section{Inertial frame of reference: the equality of resistance and impetus}

In a preliminary draft of the Definitions and Axioms section of his tract De motu (Herivel 1965), ${ }^{7}$ Newton distinguishes two senses of the concept vis insita, the precursor of our mass concept. ${ }^{8}$ These two senses of mass are resistance and impetus. They are defined thus: "[Resistance and Impetus] are distinct only in relation to each other: being resistance in so far as the body opposes itself to an

\footnotetext{
${ }^{1}$ In this paper I will use the term "falsifiability" and "falsifiable" in a deliberately loose way, that is to say, I will not be attempting to use these terms in the way Popper does.

2 See, for example, Lanczos (1970) p. 99.

3 "Based" is for the present a deliberately vague term. Its meaning depends on the epistemological interpretation given to the EIGM and my opinion on it will become clear at the end of this paper.

${ }^{4}$ In other words, the detected deviation between the two masses had been considered to fall under the accuracy error of the instruments.

${ }^{5}$ In a somewhat similar why as, for example, after the discovery of the identity of the Phosphorus and Hesperus it was no longer correct to say there are two Planets in the firmament that were demonstrated to be the same but, rather, that the same planet (Venus) manifests itself in the morning as Phosphorus, and in the evening as Hesperus.

${ }^{6}$ Of course, this does not include, for example, the balance of Eötvös, a pre-GR experiment.

${ }^{7}$ I will follow the transcription of the Manuscripts and the translations provided by Herivel (1965), pp. 315-320. The Manuscript on which I will focus in this section is the MS. Add. 3965 (5), fol. 21, fol. 26 (left half) reproduced by Herivel (1965), pp. 315-320. The distinction between resistentia and impetus is to be found in Herivel (1965, third definition, p. 318).

${ }^{8}$ In fact, Newton says that the vis insita does not differ from the inertia of matter or the mass but in our mode of conceiving. Newton conceives the mass as the quantity of matter and it is a magnitude existing by itself. The vis insita, on the contrary, is a vis, a $\delta v^{\prime} v \alpha \iota \varsigma$, and

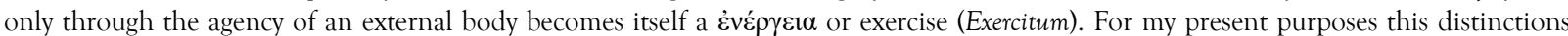
are not relevant and will not therefore be considered.
} 
impressed force, and impetus in so far as the body by yielding with difficulty attempts to change the state of the other body" (Herivel 1965, p. 315). ${ }^{9}$ That is to say, according to Newton, mass as resistance presupposes that the body is at rest and defines mass as the opposition that is practiced against another body which is attempting to change the former's state. Mass as impetus, in turn, considers that the body is moving and measures the capacity of the body to change the state of rest of another body. Of course, the velocities assigned to each of the bodies can be as diverse as we would like, but always under the restriction that the assigned velocities should observe the Galilean principle of relativity of movement. ${ }^{10}$ Accordingly, depending on the velocity assigned to each body, the role played by the different meanings of the mass will be different. The response of the body $A$, in order to maintain (perseverare) its state of rest or rectilinear uniform movement after the impact with the moving body $B$, will be explained by the resistance mass. In turn, the attempt of the body $A$ to change the state of rest or rectilinear uniform movement of body $B$ will be explained by the impetus mass. Of course, at the moment of the impact, both of these events, which have been separated for the analysis, will occur simultaneously.

Nevertheless, the meaningful and, for the purpose of my research, interesting cases of this thought experiment are the aforementioned two extreme cases. If we describe the impact of body $B$ upon body A from a frame in which body $A$ is at rest, the mass of body $A$ will be manifested as resistentia. In turn, if the inertial frame is chosen in such a way that body $A$ moves and $B$ rests, then the mass of body $A$ is considered as impetus. According to the Galilean principle of relativity of movement, rest and rectilinear uniform movement are mechanically equivalent states and, therefore, the difference between the resistentia and the impetus is merely a relative one: if the body is described from a resting frame of reference, the mass of the body will be called resistentia and if from a moving one it will be called impetus. Therefore, the differences are not those of two types of mass but rather, a difference in the manifestation of one and the same mass. The frame of reference is, at the same time, the frame of the manifestation of one and the same mass.

In my opinion, the ease and evidence with which every modern scientist accepts the Galilean principle of relativity explains not only that the difference between the aforementioned two senses of mass has disappeared from physical texts, but also the surprise that would meet any attempt to demonstrate empirically the equality between the mass resistentia and the mass impetus. ${ }^{11}$

\section{Non-inertial frame of reference: the equality of the inertial and gravitational mass}

\subsection{From the inertial to the non-inertial frame of reference}

The Galilean principle ${ }^{12}$ of relativity is part of the tendency ${ }^{13}$ to break down the notions of absolute

\footnotetext{
9 Herivel: "Resistentia et Impetus respectu solo ab invicem distincti: Resistentia quatenus corpus conatur mutare statum corporis alterius" (1965, p. 315).

${ }^{10}$ This is just what Huygens does in his De motu coporum ex perscussione (1929b) to deduce the impact laws of the rigid bodies. He uses the Gedankenexperiment of a man standing on a canoe who is bearing the two bodies hanging on his streched arms. This experiment enables him to reduce the diversity of impact cases to a basic general law.

${ }^{11}$ Such a scientist would need to carry out experiments with impacts that would have to prove that the mass of the body A when it is in rest and when it is moving with a rectilinear uniform movement is the same. Assuming the scientist follows due scientific method, he will consider any divergence of the two kinds of masses to fall within the accepted error threshold.

${ }^{12}$ The widely recognized main characteristic of the Galilean relativity principle should not prevent us from recognizing that this principle depends on or, rather, it is equivalent to the principle of the conservation of momentum. The principle of the conservation of momentum is, in turn, the postulate of the mathematization of Physics: every equation presupposes that there is no occurrence either leakage or creation of the magnitudes represented in it, and that the velocities are also variables, not constants, of the law, and that they are not fixed to any external reference system (the absolute space). Therefore, particular cases can be reduced (in the sense of the Aristotelian $\alpha$ a $v \gamma(\omega \gamma \eta)$ to the law.

13 "Tendency", whose difficulties to make its way in the history of physics are perfectly illustrated by the Leibniz-Clark correspondence. On that occasion it was Leibniz, not Newtonianism, the representative of the aforementioned tendency. It goes without saying that it is not the aim of this paper to investigate the mathematical justification of this "tendency" (see Alexander 1956).
} 
space and time in modern Physics. The state of rest and rectilinear uniform movement are different only in their relation to each other; that is to say, there is no such thing as "absolute rest" or "absolute rectilinear uniform movement". Consequently, neither is there an absolute space in respect to which a state of rest could be one of absolute rest, nor in respect to which a state of movement could be one of absolute movement. Nevertheless, there is a further possibility to rescue the idea of absolute space from its exile. If neither the uniform rectilinear movement, nor the state of rest of a body is able to demonstrate the existence of the absolute space, it would be left to uniformly accelerated movement to fulfill this task: space, in respect to which the accelerated body is accelerating, is absolute.

At first glance, this last definition of absolute space avoids the circularity from which the first definition suffers. ${ }^{14}$ In order to prove the acceleration of a body we need to refer it to an absolute space because the acceleration itself produces a visible result of its accelerated state: an endeavor, conatus, in the direction of movement.

In order to illustrate this point, let's take Newton's illustrious example; the rotating bucket full of water. ${ }^{15}$ The deformation on the shape of the water (maximum of deformation when the water is relatively at rest in the bucket) is the phenomenon with which Newton presumably demonstrated the "truly" circular motion of water. Although some might try to refute this notion of absolute space by arguing that it is possible that the entire universe could be in rotation around the bucket in the opposite direction, and therefore the movement of water might not necessarily be "true", this stance is untenable. The problem with this rebuttal is that it is focused on saving the optical appearances rather than the mechanical phenomena. It is not the impressions of two different spectators (one outside the bucket and another inside it) that we have to save but rather, the mechanical equivalence of their frames. ${ }^{16}$

Before we proceed, it is important to point out that the discussion is not about showing that Newton was wrong in his proof of absolute space. Newton's argument is perfectly valid inside the framework of inertial frame Systems. That is to say, if only inertial frame Systems were allowed, it would not be possible to avoid the Newtonian conclusion. Therefore, if we depart from the postulate of the inexistence of absolute space, we have to propose non-inertial frames of reference. The question is thus: is there any non-inertial frame of reference in respect to which the water in the bucket is at rest and the mechanical phenomena are saved? I.e. is it possible to save the mechanical phenomena if we situate the frame of reference so that it moves with the bucket? Newton says not. The GR, from a broader frame, replies that it is: we just have to imagine a body around the bucket with the sufficient gravitational attraction on the water inside the bucket to produce its observed effect.

However, what does this have to do with our initial inquiry?

\subsection{The equality of the inertial and gravitational mass: the centrifugal and the centripetal forces}

It is remarkable that the concept of centrifugal force (vis centrifuga), although it plays a central role in the demonstration of the absolute space, is rarely used by Newton in his Principia (Cohen \& Whitman 1992). ${ }^{17}$ Instead of the centrifugal force, Newton prefers to use the centripetal force to describe the rotational movement. The change in the perspective is not meaningless: from a view in which the force of the body is directed outside, Newton passes to one in which the force is directed into the center.

In order to understand this change, from the construction of the circular motion using the centrifugal force to that using the centripetal force, we have to take one step back to Huygens. In his

\footnotetext{
${ }^{14}$ Namely: absolute space is that in respect of which a resting body is at rest. But the only mean to determine whether the body is at rest is to relate it to an absolute reference frame. The same thing happens, mutatis mutandis, with a body moving with a rectilinear uniform movement.

${ }^{15}$ See Newton (1687), p. 9.

${ }^{16}$ Of course, if we assign the appropriate mass value to the universe we will be able to save also the mechanical phenomena but this will happen only, $\kappa \alpha \tau \dot{\alpha} \sigma \nu \mu \beta \varepsilon \beta \eta \kappa o ́ s$, accidentally.

${ }^{17}$ See the valuable remarks on this point by I. Cohen in Newton (1992), pp. 82-84.
} 
posthumous work, De vi centrifuga (1929a), ${ }^{18}$ Huygens quantifies the conatus of the circularly moving body for the first time. For this aim, Huygens uses a representation of a man on top of a rotating body, ${ }^{19}$ carrying with him a ball of lead that hangs on his arm. Therefore, the vi centrifuga (Huygens 1929a) is a kind of conatus that presupposes a rotational frame of reference. From the perspective of the man, the ball will point out in the radial direction as long as he is rotating with the body. Accordingly, if the man lets go of the ball, the direction of the departure of the ball is also radial. Nevertheless, Huygens is not loyal to his own presupposition and he uses both the rotating and the non-rotating frame of reference. He uses the non-rotating frame of reference in order to calculate the conatus of the tangential force, while the rotating frame of reference is used to describe the path of the ball after it has been released (Huygens 1929a, pp. 261-262). ${ }^{20}$ Each one of these uses are marked with an incongruity. Let's see why this is so.

I will begin with the second point. As I mentioned before, when it comes to describing the path of the released ball, Huygens uses a curve with its tangent perpendicular to the circle at the release point. This curve, the evolute of the circle, shows the quite problematic path that the observer in the rotating frame of reference will trace, if he were outside this frame attempting to describe the path of the released ball. The path is not simply a straight perpendicular because of the coriolis force acting on the body. The remarkable point about the evolute of the circle, as a curve proposed to describe the path of the centrifuged body, is that it considers the observer to be moving with the rotated system. Such an observer is at rest relative to the rotating body. That is to say, he thinks he is resting and therefore, he has to explain the detected centrifugal force without the aid of the rotation.

Let's turn now to the use of the tangent to the circle to represent the centrifugal force. At least since the sling experiment of Descartes, ${ }^{21}$ it has become very common to represent the supposed centrifugal force by the tangent to the circle. Is this sustainable? If we describe the circular motion from the outside, i.e., from an inertial frame relative to it, the tangential path of the movement is one of the components of the decomposition of the motion of the rotating body. At its limit, the tangential component of the acceleration is the variation of the velocity, and therefore, it equals zero in a uniform circular motion. Thus, this component of the curved motion has nothing to do with the centrifugal acceleration.

What about the force which counteracts the normal component or the centripetal force? In the case of the rotating sling, the force operates in the opposite direction to the centripetal force, and as long as the body stays in the circular path, it has the same magnitude as the centripetal force. Therefore, this is the reaction force of the Newton's third law to the centripetal force.

Let's now return to the rotating frame of reference. According to the GR, the principle of relativity should be extended to non-inertial frames of reference. This means that the same mechanical phenomenon must be able to be described from both an inertial frame of reference and from a noninertial frame of reference. Therefore, in the same manner that, according to the Galilean principle of relativity, it is not possible to demonstrate the existence of the absolute space based on the rectilinear uniform motion or rest, ${ }^{22}$ it is also not possible, according to the extended principle of relativity, to demonstrate the existence of absolute space based on accelerated motion. That means that the inertial and the non-inertial frames of reference should be mechanically equivalent. The accelerated movement of one body (considered from a non-accelerated frame of reference) must be able to be described from a non-accelerated one (i.e. from the body itself).

According to this, the same phenomenon must be able to be described from the rotating frame of reference (i.e. the frame in relation to which the rotating body is at rest) and from the inertial frame of

\footnotetext{
${ }^{18}$ I will quote from the bilingual French-Latin edition edited in Huygens (1929a).

${ }^{19}$ See Huygens: "Cogitemus maximam quampiam rotam hanc esse, ut hominem prope circumferentiam ei insistentem in $B$, facile una secum deferat; verum ita affixum ut ne excuti ipse possit; teneat autem manu sua filum cum alligata ad caput alterum fili glande plumbea" (1929a, p. 261).

${ }^{20}$ See the respective figures on p. 261 (non-rotating frame of reference) and 262 (rotating frame of reference).

${ }^{21}$ See his Principles of Philosophy, Part II § 39.

${ }^{22}$ By saying, for example, that the space in relation to which one particular rectilinear uniform movement is a rectilinear uniform movement is an absolute space.
} 
reference. As I said before, the mechanical phenomenon that we need to explain, if we are situated upon the rotating frame of reference, is the conatus directed outwards, that is to say, the centrifugal force. How can we explain the existence of this force, taking into account that the body is by assumption at rest? The answer is well-known: we have to suppose the existence of one body outside the circular path whose mass attracts the body at rest. If we suppose that the attractive force between the two bodies is the result of gravitation, then Newton's law of universal gravitation will determine their quantitative relation. In addition, due to the fact that the centrifugal force is equivalent to minus the centripetal force, by equalizing the value of the centripetal force to Newton's law of universal gravitation, we will obtain the relation between the mass of the body, whose existence has been assumed, and the distance from this body to the resting body. ${ }^{23}$ Finally, referring again to the body placed in the sling, the force of our hand pushing in the opposite direction to the centrifugal force is the reaction force, described by Newton's third law, ${ }^{24}$ to this centrifugal force.

Let's sum up the previous results.

We have shown that the conceptual difference between the centrifugal and centripetal forces is based merely on the different frame of reference in which they appear. In particular, the force that inside an inertial frame of reference appears as the centripetal force is, within the rotating frame of reference, the centrifugal force. These forces, of course, are governed by a different law. The centripetal force is governed by the second law of motion, whereas the centrifugal force is governed by Newton's law of universal gravitation. Since in each of these two laws the mass is defined differently, their names are also different: whereas the mass defined in the first law is called "inertial mass", that defined by the second law is the "gravitational mass". Nevertheless, we have shown that the differences between the forces and, therefore, between the concepts of mass, are based merely on the frame of reference. That is to say, one mass considered from an inertial frame of reference bears the name of "inertial mass" and the same mass considered from a non-inertial frame of reference is the "gravitational mass". 25 Therefore, the difference between both masses is only based on the perspective and so, their equality is that of the essence. The same mass manifests itself as inertial mass when considered from an inertial frame of reference and as gravitational mass when considered from a non-inertial frame of reference. ${ }^{26}$

\subsection{The equality of the inertial and gravitational mass: the equivalence principle}

In the previous section, I have shown one example in which different concepts of mass appear in the same physical phenomenon depending on the frame of reference from which they are described. In this new section, I will apply the same change of perspective to one well-known case, the free-falling body, the aim being to show that the equivalence principle is equivalent to the generalized principle of relativity, and to show how this equivalency works. In doing so, I will show that the equivalence principle, precisely because it is a principle, is in no way an empirically refutable proposition.

The application of different frames of reference (one inertial and the other one non-inertial) to the fall of the body takes place exactly in the opposite way to that of the centrifugal force. Whereas in the last case we departed from the inertial frame of reference and finished with the non-inertial, in the case of the free fall, we begin with the non-inertial frame of reference and end with the inertial.

\footnotetext{
${ }^{23}$ Of course, in order to obtain this relation, we have to equalize the inertial and the gravitational masses of the body and, therefore, we have to assume the main result of this paper to be accurate.

${ }^{24}$ Obviously, because we are on the rotating frame of reference, our description of the facts will not describe a rotating sling but, rather, a piece of a taught string at rest attached on the one side to the hand and, on the other side, to the body from which the description is made.

${ }^{25}$ Of course, in the generalized case, in the same way as in the case of the equality between mass as resistance and mass as impetus analyzed earlier, depending on the chosen the frame of reference we will also have intermediate cases in which the mechanical phenomenon is not completely explained neither by the inertial mass nor by the gravitational mass. In the main text, I will consider only the two extreme falls and, therefore, when I refer to the "non-inertial frame of reference" I mean the "reference system that rotates together with the body".

${ }^{26}$ Strictly speaking there is no such a thing as a mass before its appearance (where the different laws of physics are the relational frame of appearance of the mass) in the same way as there is not such a thing as a planet before its appearance, for example, as Phosphorus or as Hesperus. However, this fact, far from being an objection to our main argument, is confirmation that the equality about which we are speaking is an essential one.
} 
Let's suppose that we have a body on a table and that the table is placed on the Planet $P$. The gravitational attraction between both bodies is measured by the law of universal gravitation and depends on the gravitational masses of both bodies, their distance to each other, and the Gravitational constant. In this case, the body is at rest, i.e., our frame of reference is resting in relation to it. We are thus in the analogous situation to that of the centrifugal force, with the difference that before we posited that the existence of an external body responsible for the gravitational force was hypothetical, whereas now the existence of the Planet that attracts the body is a real one. ${ }^{27}$ The push applied by the Planet on the table, in the opposite direction to the gravitational attraction of the body, is the reaction force to this attraction.

Is this the only way to explain the physical phenomenon consisting of a body on the table? Certainly not. We can assume that there is no longer a Planet $P$, and that the table is placed inside an elevator moving upwards (in relation to the Planet that has been eliminated) with an acceleration $g$. The constant $g$ will be bigger or smaller depending on whether the mass of the Planet $P$ is bigger or smaller. The force that the table should exercise on the body, in order to move it upwards with an acceleration $g$, equals the mass of the body multiplied by $g$. In order to describe the same phenomenon as in the previous case, we have to choose a value for $g$ that equalizes the upward force of the table on the body and the previous attractive force upon the body downwards. That is to say, depending on the frame of reference we choose, the same phenomenon will appear as a body pushing a table downwards because it is being attracted by the Planet, or as a body being pushed by another body (the elevator) upwards because it is being carried upwards. The reaction forces of these forces are, on the one side, the upward force of the planet surface on the table legs and, on the other side, the downward force exerted by the cable on which the elevator is hanging from its support joint. ${ }^{28}$

As in the previous centrifugal/centripetal case, the same body, depending on whether it is considered from one frame of reference or from another, appears differently to us. In one case, it appears to us at rest, being attracted by a Planet. In the other case, it appears to us moving upwards with the acceleration $g$. In both of these cases, different concepts of mass come into play; in the former, it is gravitational mass, and in the later it is inertial mass. However, we have already seen that, according to the generalization of the Galilean principle of the relativity in the GR, the physical difference between both of these masses vanishes together with the physical difference of the frames of reference, on which the difference between the masses depends. That is to say, the affirmation of the physical equivalence of the difference between the inertial and non-inertial frame of references in the GR implies also the physical equivalence of the difference that originated in this first difference, namely, the difference between the gravitational and inertial mass.

On this basis, the structural equality between the attraction of the planet $P$ upon the body towards itself and the push of the floor of the elevator upwards implies that the value of $g$ is independent of the mass of the body. ${ }^{29}$ The next question is, what is the meaning of $g$ ?

\footnotetext{
${ }^{27}$ From the point of view of the generalized principle of relativity, the difference between real and hypothetical frames makes no sense at all. The only motive to use such a terminology is to differentiate the departing frame from the ending one. However, this distinction is misleading and I prefer not to use it. For the same reason it is also recommendable to refrain from calling the centrifugal force and, in general, the forces that arises on the rotating frame of reference as phantom or pseudo forces (in German Scheinkraft), phrases commonly in use in current handbooks.

${ }^{28}$ The analogy between the four forces of the centrifugal/centripetal case and the current one is the following (I will use the notation: A for the centrifugal case, $B$ to the centripetal case, $C$ to the present fall with the planet, $D$ without it) $A$ is to $B$ as $C$ is to $D$. The centrifugal force in $A$ is equivalent to the downward force in $C$; the reaction force of the "hand" pushing in the opposite direction to the centrifugal force in $A$ is equivalent to the surface pushing the table upwards in $C$. The centripetal force in $B$ is equivalent to the force of the floor of the elevator pushing downwards in $D$. The reaction force upon the "hand" in the direction to the body in $B$ is equivalent to the force exercised by the elevator upon the support point.

${ }^{29}$ In short. The well-known equality is: $m_{i} \cdot g=\mathrm{G} \frac{m_{g} \cdot M_{g}}{r^{2}}$ and because structurally $m_{i}=: m_{g}$ we obtain: $g=\frac{G \cdot M_{g}}{r^{2}}$ ( $m_{i}$ : the inertial mass of the body; $m_{g}$ : the gravitational mass of the body; $M_{g}$ : the gravitational mass of the Planet; $g$ : the upward acceleration of the elevator; $r$ : the distance from the center of the body to the center of the Planet; G: the gravitational constant).
} 
In order to answer this question, I will convert the dynamic state of the elevator experiment into a static one using what's known as the d'Alembert force. ${ }^{30}$ What is the picture that corresponds to the new state of static equilibrium? The answer is, of course, the free fall. The introduction of the d'Alembert force can be represented as the fixation of the support point of the elevator into a second elevator moving downwards with the velocity $g$. That is to say, the new state derived by the introduction of the d'Alembert force is represented by the cutting of the cable from which the elevator is hanging. Therefore, the elevator and, with it, the body inside it, will fall freely downwards with the acceleration $g$.

Accordingly, the acceleration of the free fall of the body is independent of its mass, because it has been shown that $g$ does not depend on the mass of the body which is falling ${ }^{31}$ and $g$ can be interpreted as the free fall acceleration of the body. This is the Galilean equivalence principle. This principle is equivalent to the equality between the inertial and gravitational masses, ${ }^{32}$ and its validity is, as the name "principle" indicates, non-empirical. I have shown that the principle of equivalence and the equality between the inertial and gravitational masses are also equivalent to the generalized principle of relativity of the reference systems. Therefore, the equality of the acceleration of different bodies ${ }^{33}$ in free fall will also be valid a priori on other Planets. ${ }^{34}$ The magnitude of $g$ will be different on different Planets, ${ }^{35}$ but it is equal for the different bodies attracted by each Planet. In short, the magnitude of $g$ is empirical and depends on each Planet but, the fact that it is equal for each Planet is a priori and not empirical. ${ }^{36}$

\section{The equality of all the four masses: resistance, impetus, inertial mass and gravitational mass}

At the end of the previous section the use of the d'Alembert force has revealed that there is a third regard with which a dynamic phenomenon can be physically described, namely, the static approach. Therefore, the addition of the d'Alembert force (also called force of inertia) reduces dynamics to static. In each of the three approaches a different concept of mass has been demonstrated: the gravitational mass (the body placed on the table), the mass as resistance (the body carried on the lift) and the mass as impetus (the body in free fall). In this division of the meaning of mass, the inertial mass has been divided into the mass as impetus and mass as resistance.

In the same way as in the case of the free fall, the d'Alembert force can also be used to describe the circular motion of the body. This approach is widely used in the handbooks ${ }^{37}$ and implies the addition of three new inertial forces to the system to obtain the equilibrium: the centrifugal force, the Coriolis force and the Euler force (this one occurs if the angular velocity vector changes in either direction or magnitude). In this new third approach the existence of the aforementioned forces is explained by the rotation of the reference system and, therefore, those forces are also called apparent forces. The mass responsible of these forces is the inertial mass, specifically the mass as impetus.

The d'Alembert principle, acting as a kind of bridge between the static and dynamic physical approach, eliminates also in some sense the strict separation between the Galilean principle of relativity and that of the GR. Due to the d'Alembert principle the absolute rest and the accelerated and

\footnotetext{
${ }^{30}$ Expressed in formulas: the initial $F=m_{i} \cdot g$ converts into $F-F^{a}=F-m_{i} \cdot g=0$, where $F=-m_{i} \cdot g$ is the d'Alembert force. We go therefore from a dynamical into a static description of the experiment. See on this point Lagrange: "si l'on imagine qu'on imprime à chaque corps, en sens contraire, le mouvement qu'il doit prendre, il est clair que le système sera réduit au repos" (1888, p. 256).

${ }^{31}$ Falling, of course, if we consider the state of the body from the point of view of d'Alembert (see d'Alembert 1743, Part II, Chap. 1).

${ }^{32}$ That is to say, the equality between the inertial and gravitational manifestations of the mass.

33 "Different" in the sense that they have different masses.

34 "Planet" in the broader sense (stars, satellites, planets, etc.).

35 "Different" in the sense that they have different masses and radii.

${ }^{36}$ Therefore, it's not necessary to perform any bizarre "experiments" such as that of David Scott (see https://nssdc.gsfc.nasa.gov/planetary/lunar/apollo_15_feather_drop.html) in order to demonstrate that two bodies with different masses will fall on the moon with the same acceleration.

${ }^{37}$ See, for example, Lanczos (1970), pp. 98-103.
} 
gravitational approaches of the same phenomenon are equivalent, making it impossible for the proponents of absolute space to defend their position. Indeed, without the application of the d'Alembert principle two relativity principles are working in the mechanics. The first one, the Galilean relativity principle, declares the mechanical equivalence between the frame of references at rest and at uniform rectilinear movement. The second one, the generalized principle of relativity, establishes the mechanical equivalence between the accelerated frame of reference and the resting frame of reference. This difference between the inertial and non-inertial systems can be used to rule out possible types of movement of the system and, therefore, to defend the existence of certain possible absolute spaces. ${ }^{38}$ Due to the reduction of the dynamics to the statics, the difference between the inertial and non-inertial systems vanishes and, as a consequence it is no longer possible to rule out these possibilities.

\section{Conclusions}

In my paper, I have shown that the difference between the inertial and gravitational mass can be reduce to, and is explained by a difference of the frame of reference. Depending on what kind of noninertial frame of reference we use to describe the mechanical phenomena the manifesting mass form will be either inertial or gravitational. The difference between both masses is a relative one (relative to the frame of reference), not an absolute one, that is not a difference of the thing itself. The same mass appears, depending on our perspective, under one aspect or another but the object itself is and remains the same. The equality that express their identity is, thus, an equality that express that the both masses are essentially the same or, rather, that there is one and the same mass which, according to the different frame of references, manifests itself in two ways. ${ }^{39}$

In order to support my position, I have detected an analogous equality expressed in the preliminary works of Newton to the Principia (Cohen \& Whitman 1992). The mass qua Resistance is to the frame of reference at rest and the mass qua Impetus to the frame of reference moving with non accelerated rectilinear moving, as the inertial mass is to the resting frame of reference and the gravitational mass is to the accelerated frame of reference. Curiously enough the response to both these statements has been materially the opposite but formally the same. In both cases the equality has been ignored; in the first case because it is too obvious, and in the second case because it is not obvious enough. Accordingly, the equality of the mass qua Impetus and qua Resistance has been assimilated as such, taking it as an essential equality. ${ }^{40}$

However, there is a second reason which also explains why the non-empirical character of EIGM has been ignored. This reason has to do with the particular unexpected but common representation of the physical phenomena, in which according to the analogy we proposed, the mass qua gravitational has to make appearance. Therefore, when a handbook comes to represent the circular motion of the body, it is standard practice to consider first the body from the inertial frame of reference and then correct it by additional terms in order consider the phenomenon from the rotating body itself. We

\footnotetext{
${ }^{38}$ For example, the existence of a non-inertial description of the facts rules out the possibility of a absolute space in relation to which our system moves with an uniform rectilinear movement.

${ }^{39}$ My conclusion can be formulated more exactly with the aid of Fregean terminology. Let's suppose that, as I show in this paper, the equality of the inertial and the gravitational mass is an equality of essence and not, say, the equality of the type "the mass of that particular body is 3,45 Kgs". One may ask how, this being so, the equality between the inertial and gravitational mass is informative at all. In other words, way is not the identity "inertial mass = gravitational mass" of the form "A = A"? Why isn't the equality between the inertial and the gravitational mass tautological? Which is the relevant information expressed by the equality that prevents us from classifying it as a tautology? The problem arises from the fact that we consider equality merely as a relation between two objects. In order to respond to such questions, Frege proposes (see his "Über Sinn und Bedeutung", 1892) the distinction between the sense (Sinn) and the reference (Bedeutung) of an expression. The Sinn is the way in which the reference is given. In our particular case, the expressions "inertial mass" and "gravitational mass" are different senses of one and the same reference. The equality is informative because it expresses not that one thing is equal to itself (in which case the equality will be tautological, and therefore non-informative) but because it express that one and the same thing (Reference) is given to us in two different ways (Senses).

${ }^{40}$ Not, of course, because the scholarly community has specifically assumed it as such equality, but rather by implication, i.e., because nobody has had the idea of empirically demonstrate the equality. In other words, the equality was so obvious that nobody (except Newton in his drafts) has recognize it as such equality.
} 
have shown that one of the added components, expressed as a force, is the centrifugal force. The point is that although we are in motion together with the rotating body and thus, the body is at rest for us, we know that we rotate (that is the reason why we added the correcting terms), and we consider the body as rotating with reference of an absolute reference system. This, of course, is far from following the generalized principle of relativity, which establishes the strict equivalence between the accelerated frame system and the gravitational field. ${ }^{41}$ In short, the common representation of the centrifugal movement and the centrifugal force uses the so called d'Alembert principle, and therefore it makes of the centrifugal force a d'Alembert force, i.e. something that is only real on a rotating frame of reference and is, therefore, strictly speaking, an "apparent force".

I have shown, therefore, two cases in which the same phenomenon are governed by different physical laws depending on the frame of reference. The mass defined inside the relation network described by the respective laws will be labeled differently. Therefore, this difference (and, also, the equality) is not accidental, i.e., empirical; rather it obeys to strictly structural arguments. The equality is thus, as Einstein suggested it in his Grundzüge der Relativitätstheorie (1969), an equality of essence. This essentiality involves, as I have shown, the non-empirical character of the constancy of the acceleration for the different bodies under a gravitational field.

Before I close this paper it is fitting to reply briefly to an objection that may be raised, not against particular aspects of my argument but, rather, against the conclusion itself. It migtht be objected that although the argument is correct, the conclusion, the non-empirical character of the equality, doesn't necessarily follow from it. In other words, it could be argued that the structural equivalence between the masses and the frames of reference is not a hindrance to its empirical character. Accordingly, the experiments to demonstrate the equality may demonstrate also the truth of the GR.

If we assume that such an objection can be rigorously upheld, ${ }^{42}$ the response would be as follows. Someone making such an objection is holding the equivalent reasoning as a hypothetical person who, after recognizing the validity of one particular geometrical proof that demonstrates that the sum of the angles of a triangle equals 180 degrees, would nevertheless aspire to corroborate or strengthen the proof by measuring the angles of empirically existing triangles: the more empirical triangles are measured the more corroborated the geometrical proof is. Obviously, nobody would take very seriously either this kind of corroboration, or the compulsion to measure that lies behind it. According to the point of view presented in this paper, the EIGM is also unable to be corroborated by the empirical data. This does not mean that EIGM is therefore false but, rather, that it belongs to a level of physical theory which is different to the empirical one. To put it using the terminology of Galileo: whereas the empirical level of the theory is to be observed using the eyes of the face, the other level, the level in which the distinctiveness of modern science is based, is to be observed using "the eyes of the soul". ${ }^{43}$ Before Einstein the only way to understand the EIGM was using the eyes of the face, but after Einstein's revolution in Physics, the EIGM can be and should be understand using the eyes of the soul. Indeed, one should not behave as if the geometrical demonstration that the sum of the angles of a triangle is 180 degrees does not yet exists.

There are many examples in the History of Sciences illustrating the aforementioned separation of levels inside the scientific discourse. Perhaps none of these illustrations is more instructive or obvious than the introduction of the principle of the conservation of mass ${ }^{44}$ by Lavoisier. ${ }^{45}$ Due to the

\footnotetext{
${ }^{41}$ The difference between our approach of the centrifugal force and the regular approach is analogous to the difference between a man placed inside an accelerating elevator who doesn't know whether he is moving or being attracted by a gravitational field, and a man who decides to take and elevator, presses the button and begins to be accelerated. In the former, only one reference frame has been used, while in the second, two frame of references are used, one (the moving one), being related to the other (the fixed one).

${ }^{42}$ This assumption is, however, unrealistic and grants too much to the opponent. It aims merely to propose a scenario in which an argument of last resort may by raised by the opponent.

${ }^{43}$ The expression "eye of the soul" goes back to Plato and was widely used by ancient and modern philosophers to refer to something in a sense equivalent to our "reason".

${ }^{44}$ The principle of the conservation of matter is also useful to illustrate that the principles of sciences are not only empirically indemonstrable but that nor are they refutable. Accordingly, if, for example, in one experiment the matter of the system at a given time A is bigger than the matter at the time $\mathrm{B}$, the conclusion would not be that the principle of the conservation of matter has been refuted but, rather: 1. that the system, supposedly close, is in reality open (it has a "hole") or 2. we are in front of a case in which the principle is
} 
introduction of this principle in his chemical research, Lavoisier could leave behind his previous blind research methodology and was able to discover (not detect) oxygen. If Lavoisier had proceed in his research career as many scientists believe that they themselves proceed,${ }^{46}$ perhaps the contemporary chemistry would continue to focus its efforts on detecting (of course, by means of very sophisticated and expensive experiments) the phlogiston.

\section{References}

Alexander, H. G. (ed.) (1956), The Leibniz-Clarke Correspondence. Together with Extracts from Newton's Principia and Opticks, Manchester: Manchester University Press.

Carr, D. (1986), "Narrative and the Real World: An Argument for Continuity", History and Theory 25(2): 117-131.

d'Alembert, J. le R. (1743), Traité de dynamique, Paris: David.

Descartes, R. (1644), Principia Philosophiae, Amsterdam: Elzevir.

Doran, R. (2013), "Editor's Introduction: Choosing the Past: Hayden White and the Philosophy of History", in Doran, R. (ed.), Philosophy of History after Hayden White, London/New Delhi/New York/Sydney: Bloomsbury, pp. $1-33$.

Einstein, A. (1969), Grundzüge der Relativitätstheorie, Braunschweig: Vieweg.

Frege, G. (1892), “Über Sinn und Bedeutung”, Zeitschrift für Philosophie und philosophische Kritik 100: 25-50.

Herivel, J. (1965), The Background to Newton's Principia, Oxford: Oxford at the Clarendon Press.

Holmes, F. L. (1998), Antoine Lavoisier. The Next Crucial Year, New Jersey: Diane Publishing Co.

Huygens, C. (1929a), De vi centrifuga, in Oeuvres complètes, Vol. 16, La Haye: Martinus Nijhoff.

Huygens, C. (1929b), De motu corporum ex percussione, in Oeuvres complètes, Vol. 16, La Haye: Martinus Nijhoff.

Lagrange, J. (1788), Méchanique Analitique, Paris: Gauthier-Villars et fils.

Lanczos, C. (1970), The Variational Principles of Mechanics, New York: Dover.

Newton, I. (1687), Philosophiae Naturalis Principia Mathematica, London: S. Pepys. (English version by I. Bernard Cohen and Anne Whitman: The Principia. Mathematical Principles of Natural Philosophy, Berkeley: University of California Press, 1992.)

subsumed under a more general principle (the transformation of matter into energy under some specific circumstances). Any attempt to demonstrate a scientific principle has to be a circular. See, for example, the circular demonstration of the law of inertia in Einstein (1969), p. 60.

${ }^{45}$ See Holmes (1998).

${ }^{46}$ That is to say, observing, and inquiring (if such a thing would be possible without using the eyes of the soul) into nature using merely the eyes of the face. 